\title{
OK Computer? A Time Analysis of Google Searches About Symptoms
}

\author{
Keith J. Petrie ${ }^{\mathrm{a}}$, Kate MacKrill ${ }^{\mathrm{a}}$, Connor Silvester ${ }^{\mathrm{a}}$, Greg D. Gamble ${ }^{\mathrm{b}}$, Nicola Dalbeth ${ }^{\mathrm{b}}$, \\ James W. Pennebaker ${ }^{c}$
}

[a] Department of Psychological Medicine, University of Auckland, Auckland, New Zealand. [b] Department of Medicine, University of Auckland, Auckland, New Zealand. [c] Department of Psychology, University of Texas, Austin, TX, USA.

Clinical Psychology in Europe, 2019, Vol. 1(2), Article 32774, https://doi.org/10.32872/cpe.v1i2.32774

Received: 2019-01-01 • Accepted: 2019-04-02 • Published (VoR): 2019-06-28

Handling Editor: Winfried Rief, Philipps-University of Marburg, Marburg, Germany

Corresponding Author: Keith J. Petrie, Psychological Medicine, Faculty of Medical and Health Sciences, University of Auckland, Private Bag 92019, Auckland, New Zealand. E-mail: kj.petrie@auckland.ac.nz

\section{Abstract}

Background: Google searches are now a popular way for individuals to seek information about the significance of common symptoms and whether they should seek medical assistance. As analysis of search patterns may help understand the demand for medical care, we examined what times over a 24-hour period and on what days of the week people searched Google for information about common symptoms.

Method: We analysed Google searches for symptoms in the United Kingdom during the week from July 30 to August 5, 2018 using Google Trends. We recorded the time points with the highest search volume for 50 common symptoms relative to other searches, and the day of the week with the highest search peak for each particular symptom.

Results: All of the peak searches for the symptoms we examined occurred during the night between 10pm and 8am. The majority 32/50 (64\%) occurred between 3am to 6am with 12/50 (24\%) between midnight and 3am. Most symptom searches were more common during the week and lowest during the weekend. Typically, searches for a particular symptom peaked at a similar time each night over the week.

Conclusions: Searches for symptoms are significantly more common during night-time hours, and particularly between 3 and $6 \mathrm{am}$. Symptom searches show relatively stable diurnal and weekly patterns.

\section{Keywords}

Google searches, symptoms, health anxiety, internet, time of day 


\section{Highlights}

- Google searches for health information are common and individuals regularly search for their specific symptoms before deciding whether to seek medical care.

- Searches for common symptoms are significantly more likely to occur, relative to other searches, during the night-time hours and are highest during the working week and lowest at weekends.

- The majority of symptom searches show relatively stable diurnal and weekly patterns.

Experiencing physical symptoms is very common but it is often difficult for individuals to determine whether the symptom is serious and needs medical attention (Pennebaker, 1982; Petrie \& Broadbent, 2019). A recent general population survey showed that individuals experience an average of five symptoms in a week, while $23 \%$ of the sample reported experiencing 10 or more symptoms (Petrie, Faasse, Crichton, \& Grey, 2014). The meaning of symptoms can often be uncertain and individuals have in the past sought advice from family and friends about whether a symptom is a sign of a serious illness (Hartzband \& Groopman, 2010). However, Google is now being used as an alternative resource for understanding symptoms, with Google searches frequently used by the public to determine the significance and threat posed by particular symptoms and whether medical assistance should be sought (Jacobs, Amuta, \& Jeon, 2017).

Perhaps it is Google's anonymity, accessibility and information availability that has seen health-related searches become the second most searched thematic area amongst all searches (Sullivan, 2016), with searches for symptoms now accounting for approximately $1 \%$ of the three billion searches each day (Pinchin, 2016). In patients specifically, a recent survey of those attending an emergency department found that $49 \%$ regularly use the internet for health information and 35\% had searched for information on their specific symptoms before presenting (Cocco et al., 2018). Patients can also check their symptoms using online symptom checking algorithms that can provide advice about whether to seek medical care (Semigran, Linder, Gidengil, \& Mehrotra, 2015).

There has been concern raised that such searches may lead to a baseless increase in health anxiety or "cyberchondria" due to the fact that searches for common symptoms are often linked to rare, serious or fatal illnesses (Filipkowski et al., 2010; North, Ward, Varkey, \& Tulledge-Scheitel, 2012; White \& Horvitz, 2009). Given the impact that health anxiety has shown in areas such as healthcare utilisation (Barsky, Ettner, Horsky, \& Bates, 2001), there is surprisingly little information available on when individuals search for symptoms and how this may relate to utilisation of health care and the demand for out of hours care. 
The access to Google search data through Google Trends has enabled research on how often particular search terms are entered relative to the total volume of searches. This data can also be aggregated for different parts of the world or different time periods. Analyses of search terms by Google Trends has been used to estimate the level of influenza illness in a population (Lampos, Miller, Crossan, \& Stefansen, 2015), stock market trends (Preis, Moat, \& Stanley, 2013), and to investigate sensitive topics like sexual behaviour, where surveys are likely to lead to misleading data (Stephens-Davidowitz, 2017). Analysis of Google Trends for symptom searches can provide aggregated data on precisely when - during the day or the week - people are more likely to be looking for information about their symptoms and thus offer information about the likely demand for nonurgent care. In this study we examined when individuals searched for common symptoms across a 24-hour period and on what days of the week. Based on previous work on the non-urgent demand for emergency department visits in the United Kingdom, which showed that the majority of non-urgent attendance occurs late at night or in the early hours of the morning (O’Keeffe, Mason, Jacques, \& Nicholl, 2018), we hypothesized that Google searches about symptoms would follow a similar pattern.

\section{Method}

\section{Symptoms}

We used a list of 50 common symptoms to investigate the peak search period for each symptom. Forty-seven symptoms were drawn from a previous study investigating the frequency of symptom complaints in a general population sample (Petrie et al., 2014). These included common symptoms such as back pain, fatigue, headache, insomnia and joint pain. We also added three other terms to the list. This included "hangover", due to the frequency of this condition reported in general population studies (Gjerde et al., 2010; Tolstrup, Stephens, \& Grønbaek, 2014) and its association with emergency room and primary care visits (Cherpitel \& Ye, 2015). As it has often been acknowledged that searching benign symptoms in Google can produce results suggesting cancer or imminent death (North et al., 2012; White \& Horvitz, 2009), we included both "cancer" and "death" in the list.

\section{Google Search Data}

Google Trends (trends.google.com) is a publicly available online tool that allows people to analyse how often a specific term or phrase has been searched in Google over a specified time period or in a particular geographic region, relative to other searches (Nuti et al., 2014). Google Trends adjusts the data by taking a random sample of searches for a term and computing its popularity relative to the total number of Google searches over 
the same period of time. The time point with highest search volume for a term has the value of 100 , while a score of 50 indicates half the popularity.

A Google Trends search for the 50 symptoms or conditions of interest in the United Kingdom was conducted. If a symptom phrase contained 'or', as in fever or increased temperature, this was changed to + in the Google Trend search. Symptoms such as back or neck pain became back pain + neck pain. Prior to searching, computer clocks were changed to London time to ensure that the Google Trend results corresponded to the correct time zone. The time period of the search was the week from July 30 to August 5, 2018. We chose this week during the summer period so the data was less likely to be affected by winter colds and flu viruses. As minutes are only available for time periods of 24-hours or less, Wednesday August 1 was taken as the representative day of the week and the time (hours and minutes) that each symptom was searched the most on this day was recorded. The day of the week that had the greatest number of searches for each symptom was also examined. Collecting data over short periods, such as the week used in the present study, has demonstrated strong predictive power and representation of future data. For example, collecting data from one Saturday-Sunday period can forecast economic trends for subsequent months (Choi \& Varian, 2012).

The rate of Google searches during the day (6am to midnight) and night were calculated using www.openepi.com and compared using a mid-P exact method. The median number of symptoms searched for on Google during the hours 6am to midnight was compared to the number at night (midnight to 6am) using the Wilcoxon non-parametric method and a Poisson regression model was to determine whether the number of Google searches performed per hour differed. Analyses were performed in SAS (v 9.4, SAS Institute Inc, Cary, NC). The significance level was set at $p<.05$.

\section{Results}

We examined the peak time for searches for each of the 50 symptoms and these are illustrated in Figure 1. All of the peak searches for symptoms occur between 10pm and 8am. Only 3 searches for symptoms (cancer, increased appetite and sexual problems) peak before midnight and only 2 (muscle pain and difficulty concentrating) are after 6am. Of the remaining symptom searches, the majority 32/50 (64\%) occur between 3am to 6am, while $12 / 50(24 \%)$ are between midnight and 3pm; these include death at $1.48 \mathrm{am}$. The hour with the most symptom searches is 4-5am with 16 (32\%) conducted during this period. 


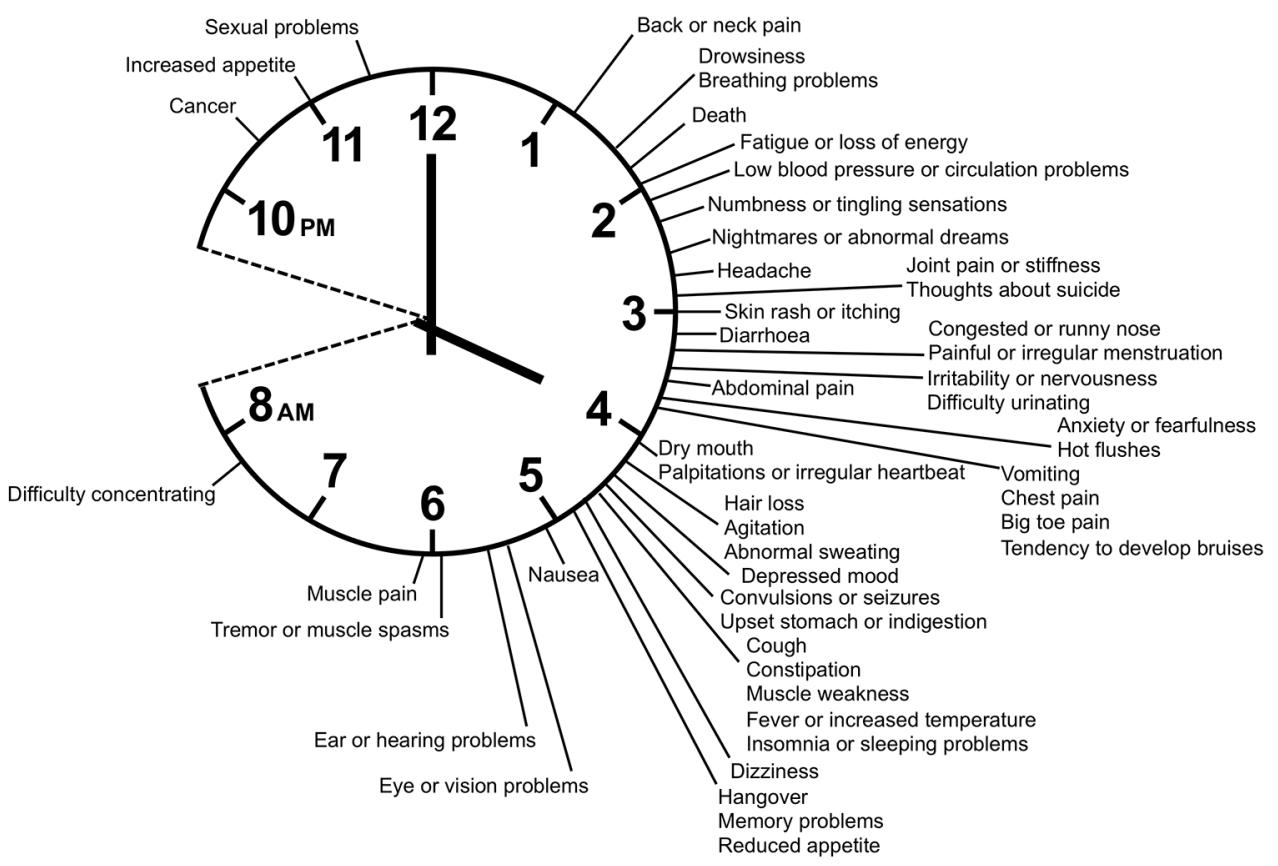

Figure 1. Peak of searches for common symptoms in United Kingdom during the week of July 30 to August 5, 2018.

In total there were 45 symptoms reported in the 6 hours from midnight to 6 am (nighttime) (7.5 searched per hour, 95\% CI [5.5, 9.9]). During the day (6am to midnight) there were 5 searches in 18 hours ( 0.28 searches per hour, 95\% CI [.01, 0.62]). There were therefore about 7 searches fewer per hour in the day than at night (rate difference -7.2 (-9.4, -5.0) (mid-P exact comparison of rates $(p<.0001)$. Significantly fewer symptoms were reported during the day than at night (median (min, max) $0(0,2)$ v $5.5(0,17)$ respectively, $p$ $=.001$ ). There was a significant difference in the number of counts observed between hour blocks (Poisson regression, $p<.0001$ ).

We next examined the distribution of symptom search peaks over the days of the week we sampled. We found that Monday, Tuesday and Friday had the greatest numbers of peaks of searches for symptoms, each with 10. Saturday was lowest with 3 symptoms, while Wednesday had 7, Thursday 4 and Sunday 6. Most symptoms (76\%) did not differ in the peak pattern of searching across the week. This is illustrated in the top two panels of Figure 2, which shows the pattern of searches for cough and vomiting. Death also shows a similar daily pattern. An exception is searches for hangover, which show a strong weekly pattern with higher levels over the weekend and lower numbers of searches for hangover symptoms during the week (bottom of Figure 2). 
Cough

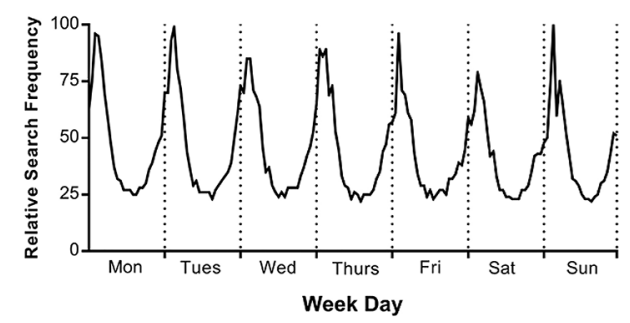

Death

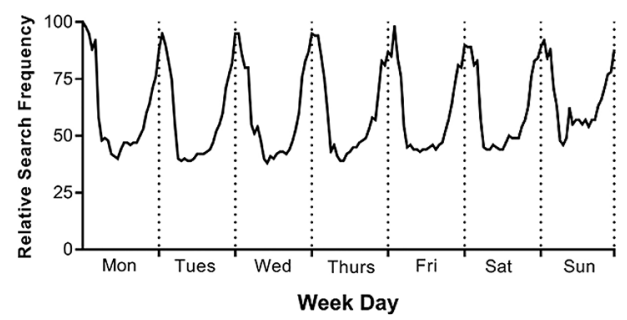

Vomiting

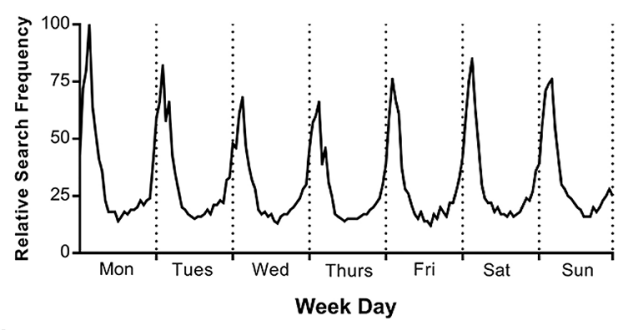

Hangover

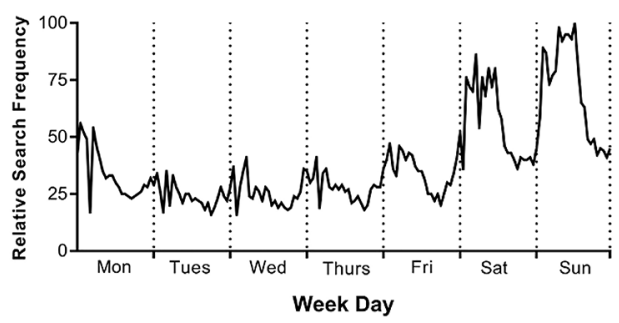

Figure 2. Weekly pattern of symptom searching for cough, vomiting, death and hangover symptoms.

The other symptoms that did not show a strong weekly pattern were increased appetite, sexual problems, low blood pressure, difficulty urinating, muscle weakness, dizziness, bruising easily, agitation, depressed mood, ear or hearing problems and difficulty concentrating.

\section{Discussion}

We found searches for symptoms are much more likely to occur relative to other searches during the night-time hours, and particularly between 3 and 6am. Most symptom searches show a consistent pattern over the week, with peaks at similar times of night. Symptom searches are more common during the week and lowest during the weekend. The results suggest that individuals with high levels of health anxiety may be advised to restrict Google symptom searches during the night-time in order to avoid unnecessary worry and healthcare use brought on by anxiety-provoking search results. Another implication may be for clinicians to ask about, and treat, sleeping problems in patients with high levels of somatic complaints.

The pattern of searches for symptoms during the night-time hours is consistent with previous research showing that people are most likely to notice symptoms when they are alone, not distracted by other activity and have time to focus on sensations that they 
rarely notice when immersed in daily life (Pennebaker, 1982). The night-time period is also a time when it is difficult to consult medical services about symptom concerns. Previous analysis of Google trends has also noted that searches for the "big" questions such as "What is the meaning of life?" and "Is there life on other planets?" also peak between 2 and 4 am (Stephens-Davidowitz, 2017). The study also highlights the use that can be made of Google Trends for understanding patient search behaviour around health issues. Analysis of Google searches has been used recently for understanding how patients manage their gout and arthritis (Jordan, Pennebaker, Petrie, \& Dalbeth, 2018), whether using Google is associated with statin intolerance (Khan, Holbrook, \& Shah, 2018) and for seeing if particular internet searches were associated with a subsequent diagnosis of pancreatic cancer (Paparrizos, White, \& Horvitz, 2016).

Some limitations of the study should be acknowledged. The data is limited to those with internet access and who use Google as opposed to other search engines. The data is from only one country and needs replication in other locations and in non-English speaking populations. As we used symptoms as specific search terms, we do not know what exactly the search was about or its context. It is also not possible to get absolute rather than relative numbers of searches. It should also be noted that there is a lack of information on how data from Google Trends is derived, including the proportion of total searches sampled and the algorithms involved (Nuti et al., 2014). Bearing these limitations in mind, it is likely that Google Trends may in the future provide more insights into how patients use the internet to seek information on health topics and as a driver for seeking health care.

It is also important to consider that Google searches may have positive effects for patients and healthcare professionals alike. For example, a recent survey of adult patients presenting to an emergency department demonstrated that searching for symptoms on Google and seeking information online resulted in a more positive doctor-patient interaction and did not reduce adherence to treatment (Cocco et al., 2018). For these patients, it may be that the internet serves as a supplementary resource that provides information that supports the doctor's opinions and enhances this relationship (Wald, Dube, \& Anthony, 2007). However, this is dependent on the information being searched for reflecting the opinion of the health professional; in cases where the two information sources are incongruent, the relationship can be negatively affected and patients may become more likely to ignore healthcare professional advice (Russ, Giveon, Catarivas, \& Yaphe, 2011). As such, while searching for symptoms may have some beneficial outcomes, the extent of this is limited by the accuracy the information searches provide.

This research has various implications for health professionals. Firstly, a greater understanding of patterns of high internet symptom searching may help health professionals better understand and determine the health anxiety levels of a patient, and how that may contribute to the experience of symptoms. Given that patients with higher anxiety levels may be more likely to misattribute general symptoms to an illness (Severeijns, 
Vlaeyen, van den Hout, \& Picavet, 2004) and are more likely to seek out information about symptoms online (Eastin \& Guinsler, 2006), asking about online search activity may be valuable clinical information.

Secondly, this research may have significant applications at a population level such as better management of healthcare services. Recent research has demonstrated that visits to health websites the preceding night can be used to predict emergency department traffic on the following day (Ekström, Kurland, Farrokhnia, Castrén, \& Nordberg, 2015). This logic may also be applied to searches for symptoms, where the number of symptoms googled may predict emergency department traffic. Further research should investigate the predictive validity of Google symptom searches, and whether deviations of such from the times and days outlined in the current study have differential implications for healthcare service traffic.

Funding: The authors have no funding to report.

Competing Interests: The authors have declared that no competing interests exist.

Acknowledgments: The authors have no support to report.

\section{References}

Barsky, A. J., Ettner, S. L., Horsky, J., \& Bates, D. W. (2001). Resource utilization of patients with hypochondriacal health anxiety and somatization. Medical Care, 39(7), 705-715. https://doi.org/10.1097/00005650-200107000-00007

Cherpitel, C. J., \& Ye, Y. (2015). Risky drinking, alcohol use disorders, and health services utilization in the US population: Data from the 2005 and 2010 national alcohol surveys. Alcoholism: Clinical and Experimental Research, 39(9), 1698-1704. https://doi.org/10.1111/acer.12801

Choi, H., \& Varian, H. (2012). Predicting the present with Google Trends. The Economic Record, 88, 2-9. https://doi.org/10.1111/j.1475-4932.2012.00809.x

Cocco, A. M., Zordan, R., Taylor, D. M., Weiland, T. J., Dilley, S. J., Kant, J., . . Hutton, J. (2018). Dr Google in the ED: Searching for online health information by adult emergency department patients. The Medical fournal of Australia, 209(8), 342-347. https://doi.org/10.5694/mja17.00889

Eastin, M. S., \& Guinsler, N. M. (2006). Worried and wired: Effects of health anxiety on informationseeking and health care utilization behaviors. Cyberpsychology \& Behavior, 9(4), 494-498. https://doi.org/10.1089/cpb.2006.9.494

Ekström, A., Kurland, L., Farrokhnia, N., Castrén, M., \& Nordberg, M. (2015). Forecasting emergency department visits using internet data. Annals of Emergency Medicine, 65(4), 436-442.e1. https://doi.org/10.1016/j.annemergmed.2014.10.008

Filipkowski, K. B., Smyth, J. M., Rutchick, A. M., Santuzzi, A. M., Adya, M., Petrie, K. J., \& Kaptein, A. A. (2010). Do healthy people worry? Modern health worries, subjective health complaints, 
perceived health, and health care utilization. International fournal of Behavioral Medicine, 17(3), 182-188. https://doi.org/10.1007/s12529-009-9058-0

Gjerde, H., Christophersen, A. S., Moan, I. S., Yttredal, B., Walsh, J. M., Normann, P. T., \& Mørland, J. (2010). Research use of alcohol and drugs by Norwegian employees: A pilot study using questionnaires and analysis of oral fluid. Fournal of Occupational Medicine and Toxicology, 5(1), Article 13. https://doi.org/10.1186/1745-6673-5-13

Hartzband, P., \& Groopman, J. (2010). Untangling the Web-Patients, doctors, and the Internet. The New England fournal of Medicine, 362(12), 1063-1066. https://doi.org/10.1056/NEJMp0911938

Jacobs, W., Amuta, A. O., \& Jeon, K. C. (2017). Health information seeking in the digital age: An analysis of health information seeking behavior among US adults. Cogent Social Sciences, 3(1), Article 1302785.

Jordan, K. N., Pennebaker, J. W., Petrie, K. J., \& Dalbeth, N. (2018). Googling gout: Exploring perceptions about gout through a linguistic analysis of online search activities. Arthritis Care \& Research, 71, 419-426. https://doi.org/10.1002/acr.23598

Khan, S., Holbrook, A., \& Shah, B. R. (2018). Does Googling lead to statin intolerance? International Journal of Cardiology, 262, 25-27. https://doi.org/10.1016/j.ijcard.2018.02.085

Lampos, V., Miller, A. C., Crossan, S., \& Stefansen, C. (2015). Advances in nowcasting influenza-like illness rates using search query logs. Scientific Reports, 5(1), Article 12760.

https://doi.org/10.1038/srep12760

North, F., Ward, W. J., Varkey, P., \& Tulledge-Scheitel, S. M. (2012). Should you search the internet for information about your acute symptom? Telemedicine fournal and e-Health, 18(3), 213-218. https://doi.org/10.1089/tmj.2011.0127

Nuti, S. V., Wayda, B., Ranasinghe, I., Wang, S., Dreyer, R. P., Chen, S. I., \& Murugiah, K. (2014). The use of Google Trends in health care research: A systematic review. PLoS One, 9(10), Article e109583. https://doi.org/10.1371/journal.pone.0109583

O'Keeffe, C., Mason, S., Jacques, R., \& Nicholl, J. (2018). Characterising non-urgent users of the emergency department (ED): A retrospective analysis of routine ED data. PLoS One, 13(2), Article e0192855. https://doi.org/10.1371/journal.pone.0192855

Paparrizos, J., White, R. W., \& Horvitz, E. (2016). Screening for pancreatic adenocarcinoma using signals from web search logs: Feasibility study and results. fournal of Oncology Practice / American Society of Clinical Oncology, 12(8), 737-744. https://doi.org/10.1200/JOP.2015.010504

Pennebaker, J. W. (1982). The psychology of physical symptoms. New York, NY, USA: Springer.

Petrie, K. J., \& Broadbent, E. (2019). Symptom perception. In C. D. Llewellyn, S. Ayers, C. McManus, S. Newman, K. Petrie, T. Revenson, \& J. Weinman (Eds.), Cambridge handbook of psychology, health and medicine (3rd ed., pp. 89-92). Cambridge, United Kingdom: Cambridge University Press.

Petrie, K. J., Faasse, K., Crichton, F., \& Grey, A. (2014). How common are symptoms? Evidence from a New Zealand national telephone survey. BMJ Open, 4(6), Article e005374.

https://doi.org/10.1136/bmjopen-2014-005374 
Pinchin, V. (2016, June 20). I'm feeling yucky :( Searching for symptoms on Google. The Keyword. Retrieved from https://blog.google/products/search/im-feeling-yucky-searching-for-symptoms

Preis, T., Moat, H. S., \& Stanley, H. E. (2013). Quantifying trading behavior in financial markets using Google Trends. Scientific Reports, 3(1), Article 1684. https://doi.org/10.1038/srep01684

Russ, H., Giveon, S. M., Catarivas, M. G., \& Yaphe, J. (2011). The effect of the internet on the patient-doctor relationship from the patient's perspective: A survey from primary care. The Israel Medical Association fournal, 13, 220-224.

Semigran, H. L., Linder, J. A., Gidengil, C., \& Mehrotra, A. (2015). Evaluations of symptom checkers for self diagnosis and triage: Audit study. British Medical fournal, 351, Article h3480. https://doi.org/10.1136/bmj.h3480

Severeijns, R., Vlaeyen, J. W. S., van den Hout, M. A., \& Picavet, H. S. J. (2004). Pain catastrophizing is associated with health indices in musculoskeletal pain: A cross-sectional study in the Dutch community. Health Psychology, 23(1), 49-57. https://doi.org/10.1037/0278-6133.23.1.49

Stephens-Davidowitz, S. (2017). Everybody lies. New York, NY, USA: Harper Collins.

Sullivan, D. (2016, May 24). Google now handles at least 2 trillion searches per year. Search Engine Land. Retrieved from http://searchengineland.com/google-nowhandles-2-999-trillion-searches-per-year-250247

Tolstrup, J. S., Stephens, R., \& Grønbaek, M. (2014). Does the severity of hangovers decline with age? Survey of the incidence of hangover in different age groups. Alcoholism, Clinical and Experimental Research, 38(2), 466-470. https://doi.org/10.1111/acer.12238

Wald, H. S., Dube, C. E., \& Anthony, D. C. (2007). Untangling the Web-The impact of Internet use on health care and the physician-patient relationship. Patient Education and Counseling, 68(3), 218-224. https://doi.org/10.1016/j.pec.2007.05.016

White, R. W., \& Horvitz, E. (2009). Experiences with web search on medical concerns and self diagnosis. AMIA ... Annual Symposium Proceedings - AMIA Symposium, 2009, 696-700.

\section{EACLIPT}

Clinical Psychology in Europe (CPE) is the official journal of the European Association of Clinical Psychology and Psychological Treatment (EACLIPT).

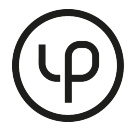

leibniz-psychology.org

PsychOpen GOLD is a publishing service by Leibniz Institute for Psychology Information (ZPID), Germany. 